\title{
Sistema integrado de gestión para las IPS de alta complejidad de la ciudad de Barranquilla basado en los estándares de acredi- tación en salud, el modelo European foundation for quality ma- nagement y el Balanced Scorecard
}

\section{Integrated management system for the high-complexity IPS of the city of Barranquilla based on the health accreditation standards, the European foundation for quality management model and the Balanced Scorecard}

\author{
Nataly Kafruni Júbiz¹, Manuel Castro Del Toro² \\ ${ }^{1}$ M.Sc. en Sistemas de Gestión. Grupo de Investigación en Gestión de la Innovación, Optimización y Medio Ambiente, ÓPTIMA. \\ Universidad Autónoma del Caribe, Barranquilla, Colombia \\ ${ }^{2}$ M.Sc. en Sistemas de Gestión. Universidad Autónoma del Caribe, Barranquilla, Colombia \\ E-mail: nataly.kafruni@uac.edu.co
}

Recibido: 24/10/17

Aceptado: 10/01/18
Cite this article as: N. Kafruni, M. Castro del Toro, "Integrated management system for the high-complexity IPS of the city of Barranquilla based on the health accreditation standards, the European foundation for quality management model and the Balanced Scorecard", Prospectiva, Vol 16, N 1, 91-99, 2018.

\section{RESUMEN}

Este artículo presenta el diseño de un Sistema Integrado de Gestión basado en los Estándares de Acreditación en Salud, el EFQM (European Foundation for Quality Management) y el BSC (Balance Scorecard), con el fin de intervenir las necesidades y problemáticas que se presentan durante la prestación del servicio en las Instituciones Prestadoras de Salud de alta complejidad de la ciudad de Barranquilla. Se aplicó como instrumento de recolección de la información, una encuesta dirigida a quince (15) IPS, la cual permitió conocer el estado de gestión de la muestra seleccionada, identificando que a pesar de que se evidencia en la mayoría de las IPS, el compromiso gerencial con la planeación estratégica y la importancia del mejoramiento continuo, una de las principales falencias que se presentan, es el debilitamiento del factor Recursos Humanos, evidenciando la poca motivación y capacitación de los colaboradores. Con la articulación de los tres modelos de gestión se logró obtener una herramienta de gestión efectiva que permite evaluar la gestión integral de la organización, tanto administrativa como operativamente, y de esta forma mejorar la prestación del servicio y garantizar la satisfacción del cliente.

Palabras clave: Acreditación en salud; Balance Scorecard; Competitividad; EFQM; Estrategia; Factores Claves de Éxito; ISO 9001.

\section{ABSTRACT}

This article presents the design of an Integrated Management System based on the Health Accreditation Standards, the EFQM (European Foundation for Quality Management) and the BSC (Balanced Scorecard), in order to aid the needs and problems that arise during the service of high complexity health institutions in the city of Barranquilla. A survey of fifteen (15) IPSs was used as an information collection tool, which allowed us to know the management status of the selected sample. More into details, the survey showed that in spite of the IPSs commitment with strategic planning and continuous improvement, there was a weakening of the Human Resources factor, evidencing the lack of motivation and training of employees. With the articulation of the three management models we were able to obtain effective management tool that assesses the management of the organization, both administratively and operationally. Thus, improving the service delivery and ensuring customer satisfaction.

Key words: Accreditation in health; Balanced Scorecard; Competitiveness; EFQM; Strategy; Key Success Factor; ISO 9001. 


\section{INTRODUCCIÓN}

El entorno competitivo y cambiante en el que se mueven las organizaciones, exige altos niveles de gestión que permitan analizar el desempeño de los procesos y si se cuentan con herramientas que posibiliten evaluar la efectividad del sistema organizacional [1].

Específicamente, el sector salud se constituye en uno de los pilares de la ciudad de Barranquilla, teniendo en cuenta el servicio que le brinda a la comunidad y las características especiales del mismo, con una capacidad instalada de 568 IPS de las cuales el 5,98\% son de alta complejidad [2].

Este artículo pretende abordar los diferentes factores que deben considerase en la implementación de Sistemas Integrados de Gestión efectivos, por parte de las empresas del sector salud, particularmente, las IPS de alta complejidad, con el objeto de dar solución a las principales problemáticas que se presentan en la prestación del servicio en cuanto a calidad, oportunidad y tiempo de atención.

Por tanto, a pesar de que un Sistema de Gestión promueve una fuerte orientación al cliente, las empresas deciden adoptarlo principalmente por el convencimiento propio de la mejora que les aportará dicho sistema [3]; es así, como los modelos de gestión permiten a las empresas lograr una mejora significativa en la satisfacción de los clientes, los empleados y los resultados empresariales, a través de su capacidad para alcanzar mejoras en las actividades [4].

El presente artículo analiza este contexto y propone evaluar el desempeño de los procesos a lo largo del tiempo y observar las tendencias que no lleven a gestionar de forma efectiva los riesgos que puedan obstaculizar el desarrollo de las estrategias y por consiguiente el logro de los objetivos estratégicos, a través del diseño de un Sistema Integrado de Gestión, fundamentado en los Estándares de Acreditación en Salud, el modelo European Foundation for Quality Management y el Balanced Scorecard.

\section{METODOLOGÍA}

La metodología utilizada fue de tipo cualitativo, ya que a partir de la indagación del tema de estudio, se buscó comprender e interpretar desde de la información recolectada [5], los procesos, los procedimientos y los factores claves que inciden en el éxito de las IPS de alta complejidad de la ciudad de Barranquilla.

Así mismo, se diseñó un nuevo sistema integrado de gestión, que promueve ventajas competitivas en el sector de la salud, buscando dar solución a las problemáticas de calidad, tiempo de atención y oportunidad.
De la mano, el método de investigación deductivo [6], considerando que el estudio se basó en el Modelo de Excelencia EFQM, la herramienta organizacional Balanced Scorecard y los Estándares de Acreditación en Salud, caracterizados por ser modelos universales mediante los cuales se buscó dar solución a la problemática planteada. Además, se involucraron características del método descriptivo-explicativo buscando tener una visión amplia y confiable sobre la situación real del sector salud.

\section{RESULTADOS Y DISCUSIÓN}

Una vez realizado el diagnóstico a las IPS de alta complejidad de la ciudad de Barranquilla, se generaron una serie de elementos que integraban los tres sistemas referenciados con anterioridad; fundamentado en las experiencias, revisión documental y observación de quince (15) IPS certificadas con el modelo ISO 9001, lo anterior permitió identificar el estado de gestión de las mismas, así como las deficiencias que obstaculizan el logro de los objetivos organizacionales definidos por cada una de ellas.

Se espera que las instituciones propendan por la productividad y el mejor aprovechamiento de los recursos disponibles, que en pocas palabras conlleva a mejores resultados en aspectos administrativos, por tanto el EFQM propone un sistema de gestión cimentado en procesos de mejora continua [7]. En la tabla 1 se muestran las categorías evaluadas.

Tabla 1. Categorías evaluadas.

Table 1. Evaluated categories.

\begin{tabular}{|c|c|c|}
\hline CATEGORÍAS & SUBCATEGORÍAS & ÍTEM \\
\hline Direccionamiento & $\begin{array}{c}\text { Gestión y direccionamiento } \\
\text { organizacional }\end{array}$ & $\begin{array}{l}\text { Misión, Visión, Valores } \\
\text { organizacionales }\end{array}$ \\
\hline \multirow{2}{*}{ Gerencia } & \multirow{2}{*}{$\begin{array}{l}\text { Alineación estratégica } \\
\text { Factores claves }\end{array}$} & $\begin{array}{c}\text { Materialización de } \\
\text { planeación estratégica }\end{array}$ \\
\hline & & $\begin{array}{l}\text { Rendimiento final } \\
\text { planificado }\end{array}$ \\
\hline \multirow{2}{*}{$\begin{array}{l}\text { Recursos } \\
\text { Humanos }\end{array}$} & \multirow{2}{*}{$\begin{array}{c}\text { Gestión de } \\
\text { Recurso Humano }\end{array}$} & $\begin{array}{l}\text { Plan de formación, } \\
\text { clima laboral }\end{array}$ \\
\hline & & $\begin{array}{l}\text { Satisfacción de empleados, } \\
\text { Medidas de percepciones }\end{array}$ \\
\hline \multirow{2}{*}{$\begin{array}{l}\text { Gestión de } \\
\text { Tecnología }\end{array}$} & \multirow{2}{*}{$\begin{array}{c}\text { Gestión de compras } \\
\text { Tecnología }\end{array}$} & Gestión de proveedores \\
\hline & & $\begin{array}{l}\text { Adquisición-entrenamiento } \\
\text { (Plataformas y equipos) }\end{array}$ \\
\hline $\begin{array}{l}\text { Sistemas de } \\
\text { Información }\end{array}$ & Sistemas comunicación & $\begin{array}{l}\text { Gestión de información y } \\
\text { conocimiento }\end{array}$ \\
\hline \multirow{2}{*}{$\begin{array}{l}\text { Integración en } \\
\text { Redes }\end{array}$} & \multirow{2}{*}{ Conectividad de redes } & Difusión de la información \\
\hline & & Desarrollo de la estructura \\
\hline \multirow{2}{*}{ Ambiente Físico } & \multirow{2}{*}{ Infraestructura } & Mejoramiento permanente \\
\hline & & Evaluación de riesgo \\
\hline $\begin{array}{l}\text { Gestión } \\
\text { Asistencial }\end{array}$ & Prestación del servicio & $\begin{array}{l}\text { Planeación, Control, Gestión } \\
\text { clínica, Evaluación, Servicio }\end{array}$ \\
\hline
\end{tabular}


A continuación se describen en forma general los resultados obtenidos y el análisis de los mismos. Para ello se analizaron las respuestas diseñadas por categoría. Se muestra el resultado general, teniendo en cuenta, el valor promedio de cada criterio, llegando a intervenir en ella como punto de partida y de refuerzo para el desarrollo del sistema.

Cabe resaltar que en el presente estudio, se tomó como base la escala Likert, en la cual los elementos se evalúan de uno a cinco (1-5), donde uno es deficiente, dos es insuficiente y tres es aceptable, mientras los valores cuatro y cinco son sobresaliente y excelente respectivamente; se analiza como límite inferior de evaluación un puntaje de tres (3), es decir, se manejan los promedios $\mathrm{y} /$ o puntajes iguales o inferiores al mismo como puntos susceptibles de mejora (si bien cualquier punto que no alcance una ponderación de cinco (5), será considerado como tal, estos toman mayor preponderancia para la generación de valor al momento de generar la propuesta de sistema integrado) dentro de cada organización o en términos globales, dentro del sector estudiado.

En la figura 1, se puede notar que los puntos álgidos sobre los cuales debe hacerse hincapié para el fortalecimiento del sistema, son los criterios de Recursos Humanos y Ambiente Físico, sin dejar de lado los otros criterios.

Figura 1. Gráfico del Diagnóstico general IPS alta complejidad. Figure 1. Diagram of General Diagnostics IPS High Complexity.

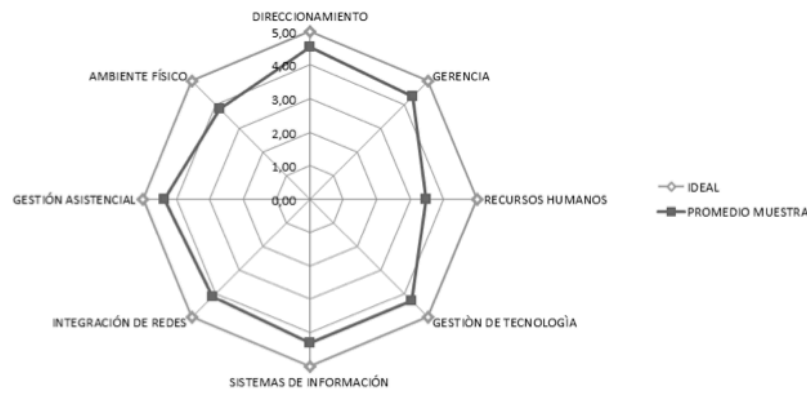

Se puede apreciar como el promedio de la muestra estudiada se acerca en algunos criterios a su puntaje máximo, tomando esto como un hecho de carácter esperado debido a que las mismas poseen sistemas de calidad que les han permitido fortalecer aspectos organizacionales, sin embargo, durante el estudio realizado se pudo notar cierto nivel de subjetividad al momento de autoevaluarse; más aún, teniendo en cuenta esto, se obtuvieron puntajes en criterios claves para el engranaje de las organizaciones que ponen en evidencia el debilitamiento del funcionamiento holístico de las IPS. Para este caso práctico los promedios más bajos y por debajo del nivel aceptable, son los correspondientes a los criterios de Ambiente Físico y Recursos Humanos con promedios de tres coma ochenta y dos $(3,82)$ y tres coma cuarenta y seis $(3,46)$.
Teniendo en cuenta lo anterior, se puede manifestar que al fallar en puntos clave como los ya mencionados, se puede llegar a afectar de manera negativa los demás criterios evaluados.

\subsection{Factores claves del éxito}

Tomando como fundamento práctico la experiencia de las IPS de alta complejidad sobre las cuales se realizó el diagnóstico y como criterio teórico acerca de cómo un BSC debidamente cimentado debe formar una serie de objetivos y medidas vinculadas [8], que son a la vez consistentes y mutuamente reforzantes, lo anterior se convierte en factores de éxito para las organizaciones que apliquen este tipo de gestión. De esta manera, a continuación se esbozan los factores claves de éxito más determinantes en el desarrollo de estrategias efectivas de las organizaciones estudiadas.

En primera instancia la Perspectiva Financiera brinda el enfoque global sobre el cual se establecen las demás estrategias organizacionales, es decir, los objetivos y estrategias; es así como se define como factor clave en dicha perspectiva, la consecución de una estructura financiera óptima (inversión) que dé como resultado la rentabilidad del negocio. Así mismo, el aumento de la cuota de mercado y la satisfacción de los clientes, son punto de partida en cada análisis realizado durante el diagnóstico incurriendo entonces en la Perspectiva Clientes, los métodos y estrategias que se utilizan para lograr fidelización de usuarios y la captación de otros nuevos son factores claves para las organizaciones [9].

Tomando el mismo enfoque, las organizaciones manifiestan como eje central de estos factores el mejoramiento continuo de los procesos, mediante la implementación de nuevas tecnologías o sistemas que propendan por el cumplimiento de requerimientos, llevando así a la Perspectiva Procesos a su punto clave mediante la construcción de servicios de calidad [10].

Por último, el estudio arroja como punto común en la Perspectiva de Aprendizaje y Crecimiento un factor importante debido a los resultados obtenidos como promedio en el criterio de Recurso Humano. Las organizaciones se centran en esta perspectiva otorgándole total valor a las competencias del personal y su capacidad de liderazgo, como requisito para lograr motivación e innovación del recurso.

\subsection{Mapa conceptual del modelo}

En primera instancia se muestra el mapa conceptual con todos los componentes tanto del modelo de gestión en salud como la herramienta estratégica y los criterios organizacionales que se implementaron para el sistema integrado.

Posteriormente, se muestra de manera general, como los Estándares de Acreditación en Salud, los criterios del 
EFQM y las perspectivas del BSC se relacionan, haciendo un primer análisis de su interacción y alineación.

El mapa conceptual para el modelo de gestión propuesto que se ilustra en la figura 2, involucra los criterios y elementos integradores del Sistema de Gestión de la Calidad planteados, de tal forma que se logre obtener mediante esta articulación una dirección y alineación estratégica que lleve al cumplimiento de la misión de la organización y al mejoramiento de la prestación del servicio en el sector salud.

Figura 2. Mapa conceptual: sistema integrado de gestión para las IPS de alta complejidad.

Figure 2. Conceptual map: high complexity "IPS" integrated management system.

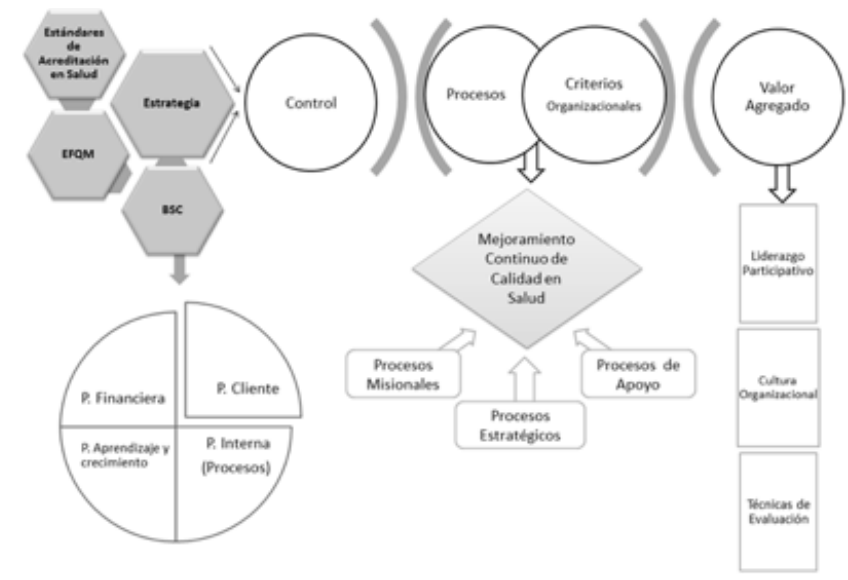

La figura 2 muestra como la articulación de los tres modelos de gestión propuestos, permitirá llevar a cabo una mejor gestión en las IPS de alta complejidad a través de implementación de un sistema integrado que propenda por el mejoramiento continuo de la calidad en salud y la transformación de la cultura organizacional, lo cual se traducirá en valor agregado para las instituciones.

A continuación se ilustran en la figura 3, las alineaciones correspondientes entre los modelos de gestión y herramientas estratégicas propuestas.

Figura 3. Integración de los Estándares de Acreditación en Salud, el EFQM y el BSC. Figure 3. Integration of Health Accreditation Standards, EFQM and BSC.

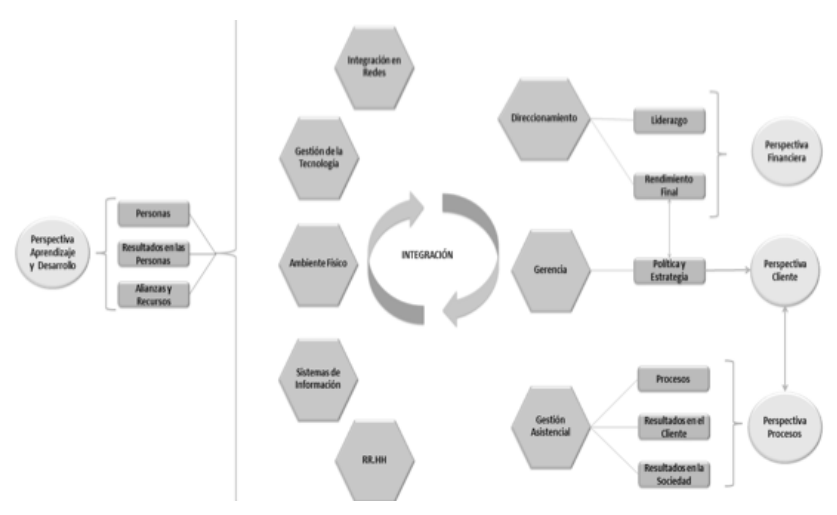

Según lo planteado, se identifica que todos los criterios del EFQM y las perspectivas del BSC se encuentran contemplados en los Estándares de Acreditación en Salud, ya sea de manera implícita o explícita.

A partir del análisis comparativo realizado, se establece la relación del modelo EFQM y el BSC con los Estándares de Acreditación en Salud y se identifican elementos comunes como se explica a continuación.

Teniendo en cuenta que las Instituciones Prestadoras de Servicios de Salud juegan un papel preponderante en la satisfacción de las necesidades crecientes de atención en salud que demanda la población, situación que exige un direccionamiento y una gerencia efectiva donde el liderazgo, la innovación, la adaptación al cambio y el mejoramiento continuo de la calidad deben convertirse en elementos propios de su cultura como estrategias de competitividad, se presenta la siguiente alineación de los elementos comunes entre los tres modelos de gestión propuestos en la siguiente investigación [11].

En primera instancia, se identifica que la perspectiva financiera del BSC, y los criterios liderazgo y rendimiento final del EFQM, se encuentran inmersos en el Estándar de Acreditación en Salud Direccionamiento a partir de la definición de la misión y visión de la empresa y del rendimiento que consigue la misma como resultado de dicha planificación. Dichos aspectos influyen en el proceso de toma de decisiones, el comportamiento del usuario como individuo y como grupo y la manera en que se consiguen los objetivos planteados por la organización [12].

Bajo el mismo enfoque estratégico, los criterios, política y estrategia y rendimiento final, del EFQM, se encuentran alineados de manera explícita con el Estándar de Gerencia al definir cómo materializa la organización su planeación estratégica mediante una estrategia claramente definida ejecutada a través de actividades que permitirán alcanzar los objetivos propuestos.

Implícitamente las perspectivas financiera y de procesos del BSC, al desarrollar el mapa estratégico, se articulan bajo las orientaciones de la misión, la visión y los valores y el análisis del comportamiento de indicadores financieros [13].

Esta articulación permite que la institución describa cómo cada unidad funcional identifica sus clientes y cómo la gerencia responde a sus necesidades apoyada en la monitorización y el mejoramiento de calidad [14]. También puntualiza cómo se involucran las unidades funcionales en la construcción del plan estratégico, cómo se implementa y se despliega dicho plan en las áreas y cómo se definen las políticas respecto de los servicios a proveer [3]. 
Como parte de los procesos misionales, los Estándares de Acreditación en Salud Asistenciales establecen el modelo de prestación de servicio basado en niveles superiores de calidad que satisfagan las necesidades de los clientes. En el caso del EFQM los criterios procesos, resultados en los clientes y en la sociedad, definen que tanto el modelo como la herramienta consideran que hay que incidir en los procesos para lograr una adecuada satisfacción del cliente. El BSC, desde sus perspectivas de clientes y procesos analiza la adecuación de los procesos y la satisfacción de los clientes para el logro de la planeación estratégica y el rendimiento financiero y organizacional.

En este sentido, se garantiza la prestación de un servicio seguro y confiable que permita obtener resultados en salud, respondiendo a las exigencias del sector, aumentando la eficiencia organizacional y administrativa en la reducción de costos.

De otro lado, el Estándar Gestión de la Tecnología se integra con el criterio alianzas y recursos del EFQM, al planificar y gestionar el funcionamiento eficaz de los procesos a partir de la adquisición de la tecnología requerida para prestar una atención segura. Se complementan con la perspectiva del BSC aprendizaje y desarrollo, a través de la habilidad que tiene la organización para mejorar y aprender favoreciendo la competitividad en su entorno.

A partir de la identificación de las necesidades de compra o renovación de tecnología, se pueden mejorar procesos y servicios generando valor agregado y satisfaciendo las expectativas del cliente y el éxito del negocio.

Con respecto al Estándar de Recursos Humanos, se resalta a la persona como el talento que hace posible que las organizaciones funcionen. Son estas, las encargadas de controlar la calidad, diseñar, producir, distribuir los productos y servicios, así como de establecer sus objetivos y estrategias. Sin gente eficiente es imposible que una organización logre sus objetivos.

Bajo esta premisa, los criterios personas y resultados en las personas del EFQM, plantean que los trabajadores son importantes para la gestión de la calidad; en el caso del BSC, bajo la perspectiva de aprendizaje y desarrollo, para el logro de la misión y visión de la organización.

Por otra parte, el Estándar Sistemas de Información, determina los elementos sustanciales de la organización para el diseño y puesta en marcha de un proceso coherente de gerencia de la información y de los recursos utilizados para su adecuado desarrollo. Para el caso del criterio alianzas y recursos del EFQM se gestiona la información y los conocimientos que apoyan el fun- cionamiento eficaz de los procesos; en el caso del BSC, bajo la perspectiva de aprendizaje y desarrollo, se definen los sistemas que proveen de esta información útil para el trabajo.

De esta manera, la organización define los requerimientos de información relacionados con los procesos de atención y necesidades de los clientes, la planeación, direccionamiento y mejoramiento de la organización, la gestión de recursos y la productividad. La articulación de estas herramientas de gestión permite describir como se promueve la toma de decisiones sobre hechos y datos y su acceso para el análisis que permita monitorear y referenciar de manera sistemática el proceso [4].

En torno al Estándar Ambiente Físico, se define el mejoramiento continuo de la infraestructura y se establece una serie de procesos y políticas encaminados a generar mecanismos operativos de prevención y manejo de los riesgos potenciales, con el fin de garantizar la seguridad y calidad del entorno donde se desarrollan los procesos organizacionales.

Para el caso del criterio alianzas y recursos del EFQM se gestiona la infraestructura necesaria y los materiales, que apoyan el funcionamiento eficaz de los procesos; en el caso del BSC se alinea con el enfoque de mejoramiento de la operación continua, que incluye la seguridad en las instalaciones y del colaborador minimizando los riesgos asociados a la actividad laboral. Es de vital importancia que la organización defina e implemente procesos que gestionen de manera eficiente los recursos físicos y el mantenimiento de la seguridad en las instalaciones.

Acerca del Estándar Integración en Redes, se toma como base la articulación y conectividad de la empresa con su entorno competitivo. En el caso del EFQM se complementa con el criterio alianzas y recursos como parte de la colaboración externa que tiene la empresa con su mercado; en el caso del BSC, bajo la perspectiva de aprendizaje y desarrollo, se integra con la generación de nuevo conocimiento que favorece la innovación y competitividad en su entorno.

De acuerdo a lo anterior, se determina de manera conceptual la integración de los tres modelos de gestión propuestos permitiendo identificar la articulación de estos enfoques que servirá como herramienta fundamental de gestión en el ámbito estratégico de las IPS de alta complejidad de ciudad de Barranquilla.

\subsection{Sistema integrado de gestión}

Los sistemas de gestión se constituyen en la estrategia que puede implementar las pequeñas y medianas empresas y que se convierte en el patrón a seguir por la misma [15], por lo cual se establece que para ser com- 
petitivos, es necesario identificar un sistema de gestión apropiado para su organización. Para identificarlo, los directivos de las Pymes deben tener claro cuál es el propósito que pretenden alcanzar por medio de su gestión.

Con el fin de crear ventajas competitivas para la organización y contribuir a un desarrollo sostenible, un Sistema Integrado de Gestión tiene que ser amplio y debe incluir la cadena de producción en su totalidad como también las partes interesadas [16].

Las empresas que operan un Sistema Integrado de Gestión obtienen beneficios tales como el ahorro de recursos, una mejor utilización de estos y la mejora de la comunicación en toda la organización. Sin embargo, para que los beneficios se materialicen, es esencial que las organizaciones sean conscientes de los retos y obstáculos que acompañan a la integración de los sistemas [17]. Si estos problemas no se tratan temprano se puede retrasar el desarrollo del proceso de integración [18].
Una manera de integrar modelos es implementar un cuadro de mando integral que permita a los directivos tener un instrumento de control que despliegue la estrategia y lleve a la empresa al éxito, y por otro lado la implementación de un sistema de calidad que a partir de la situación actual de la organización, pretende conseguir mejoras, optimizando el funcionamiento de la empresa, y así manejar la parte operativa [19].

La implementación de sistemas integrados de gestión permite mejorar las prácticas y capacidades de la empresa, facilita la comunicación y sirve como técnica para entender y gestionar el resultado, la planificación y la formación [20].

Teniendo en cuenta cada uno de los Estándares de Acreditación en Salud, los criterios propuestos por el EFQM, las perspectivas del BSC [8], se estableció la articulación entre los mismos tal como se presenta en la tabla 2.

Tabla 2. Alineación de requisitos.

Table 2. Requirement integration.

\begin{tabular}{|c|c|c|c|}
\hline ESTÁNDAR DE ACREDITACIÓN EN SALUD & CRITERIO DEL EFQM & PERSPECTIVA DEL BSC & PROCESOS IDENTIFICADOS \\
\hline \multirow[t]{2}{*}{ Asistenciales } & $\begin{array}{l}\text { Procesos } \\
\text { - Diseño y gestión sistemática de los procesos } \\
\text { - Introducción de las mejoras necesarias en los pro- } \\
\text { cesos mediante la innovación, a fin de satisfacer ple- } \\
\text { namente a grupos de interés. } \\
\text { - Diseño y desarrollo de productos y servicios de acu- } \\
\text { erdo a las necesidades y expectativas de los clientes. } \\
\text { - Acompañamiento y seguimiento al cliente posterior a } \\
\text { la prestación del servicio. } \\
\text { - Gestión y mejora de las relaciones con los clientes. }\end{array}$ & $\begin{array}{c}\text { Perspectiva Cliente } \\
\text { Perspectiva } \\
\text { Procesos Internos }\end{array}$ & $\begin{array}{l}\text { - Agenda Médica } \\
\text { - Admisiones } \\
\text { - Consulta Externa } \\
\text { - Imágenes Diagnósticas } \\
\text { - Cirugía } \\
\text { - Apoyo Diagnóstico } \\
\text { - Urgencias } \\
\text { - Hospitalización } \\
\text { - Unidad de Cuidados Intensivos }\end{array}$ \\
\hline & $\begin{array}{l}\text { Resultados en los clientes } \\
\text { - Medidas de percepción } \\
\text { - Indicadores de rendimiento } \\
\text { Resultados en la sociedad } \\
\text { - Medidas de percepción } \\
\text { - Indicadores de rendimiento }\end{array}$ & & \\
\hline Integración en Redes & $\begin{array}{l}\text { Alianzas y recursos } \\
\text { - Gestión de las alianzas externas. } \\
\text { - Gestión de los recursos económicos y financieros. }\end{array}$ & $\begin{array}{c}\text { Perspectiva Aprendizaje } \\
\text { y Desarrollo }\end{array}$ & - Direccionamiento y Gerencia \\
\hline Direccionamiento & $\begin{array}{l}\text { Liderazgo } \\
\text { - Desarrollo de la misión, visión y valores } \\
\text { - Compromiso personal con el desarrollo, puesta en } \\
\text { práctica y mejora continua } \\
\text { - Implicación de los líderes de área } \\
\text { - Motivación, apoyo y reconocimiento a los colabora- } \\
\text { dores de la organización. } \\
\text { Resultados clave } \\
\text { - Resultados claves. } \\
\text { - Indicadores claves. }\end{array}$ & Perspectiva Financiera & $\begin{array}{l}\text { - Direccionamiento y Gerencia } \\
\text { - Garantía de la calidad y } \\
\text { auditoría } \\
\text { - Gestión Financiera } \\
\text { - Dirección Científica } \\
\text { - Gestión Comercial }\end{array}$ \\
\hline Gerencia & $\begin{array}{l}\text { Política y estrategia } \\
\text { - Política y estrategia fundamentadas en necesidades } \\
\text { y expectativas actuales y futuras. } \\
\text { - La información procedente de las actividades } \\
\text { relacionadas con la medición del rendimiento, inves- } \\
\text { tigación, aprendizaje y creatividad son el fundamento } \\
\text { de la política y estrategia. } \\
\text { - El desarrollo, revisión y actualización de la política y } \\
\text { estrategia del servicio. } \\
\text { - La política y estrategia se comunican y despliegan } \\
\text { mediante un esquema de procesos claves. }\end{array}$ & $\begin{array}{c}\text { Perspectiva } \\
\text { Procesos Internos } \\
\text { Perspectiva Financiera }\end{array}$ & $\begin{array}{l}\text { - Direccionamiento y Gerencia } \\
\text { - Garantía de la calidad y } \\
\text { auditoría } \\
\text { - Gestión Financiera } \\
\text { - Gestión Comercial } \\
\text { - Dirección Científica }\end{array}$ \\
\hline
\end{tabular}




\begin{tabular}{|c|c|c|c|}
\hline ESTÁNDAR DE ACREDITACIÓN EN SALUD & CRITERIO DEL EFQM & PERSPECTIVA DEL BSC & PROCESOS IDENTIFICADOS \\
\hline Recursos Humanos & $\begin{array}{l}\text { Gestión del personal } \\
\text { - Planificación, gestión y mejora del talento humano. } \\
\text { - Identificación, desarrollo y mantenimiento del cono- } \\
\text { cimiento y las capacidades de los colaboradores. } \\
\text { - Delegación de funciones a los colaboradores y } \\
\text { autonomía } \\
\text { - Comunicación permanente con los colaboradores } \\
\text { - Política de estímulo y reconocimiento al trabajo } \\
\text { Resultado de las personas } \\
\text { - Medidas de percepción } \\
\text { - Indicadores de rendimiento }\end{array}$ & $\begin{array}{l}\text { Perspectiva Aprendizaje } \\
\text { y Desarrollo }\end{array}$ & $\begin{array}{l}\text { - Gestión del Talento Humano } \\
\text { - RSE }\end{array}$ \\
\hline Gestión de la Tecnología & $\begin{array}{l}\text { Alianzas y recursos } \\
\text { - Gestión de la Tecnología. }\end{array}$ & $\begin{array}{l}\text { Perspectiva Aprendizaje } \\
\text { y Desarrollo }\end{array}$ & $\begin{array}{l}\text { - Gestión de la Tecnología } \\
\text { - Dirección Científica }\end{array}$ \\
\hline Sistemas de Información & $\begin{array}{c}\text { Alianzas y recursos } \\
\text { - Gestión de la información y del conocimiento. }\end{array}$ & $\begin{array}{l}\text { Perspectiva Aprendizaje } \\
\text { y Desarrollo }\end{array}$ & $\begin{array}{l}\text { - Gestión de la Información } \\
\text { - Dirección Científica }\end{array}$ \\
\hline Ambiente Físico & $\begin{array}{c}\text { Alianzas y recursos } \\
\text { - Gestión de edificios, equipos y materiales. }\end{array}$ & $\begin{array}{l}\text { Perspectiva Aprendizaje } \\
\text { y Desarrollo }\end{array}$ & $\begin{array}{l}\text { - RSE } \\
\text { - Ambiente Físico } \\
\text { - Compras }\end{array}$ \\
\hline
\end{tabular}

De acuerdo a lo anterior, se presenta la relación de los procesos identificados de la interacción entre los Estándares de Acreditación en Salud, los criterios del EFQM y las perspectivas estratégicas del BSC. Esto se constituye en la base para la elaboración del mapa de procesos propuesto para el Sistema Integrado de Gestión dirigido a las IPS.
En la figura 4, se presenta la alineación entres los procesos y los temas estratégicos del BSC, que se considerará en el diseño de la estructura general del mapa de procesos modelo.

Figura 4. Alineación entres los procesos y los temas estratégicos del BSC.

Figure 4. Alignment between the strategic processes and issues of the BSC.
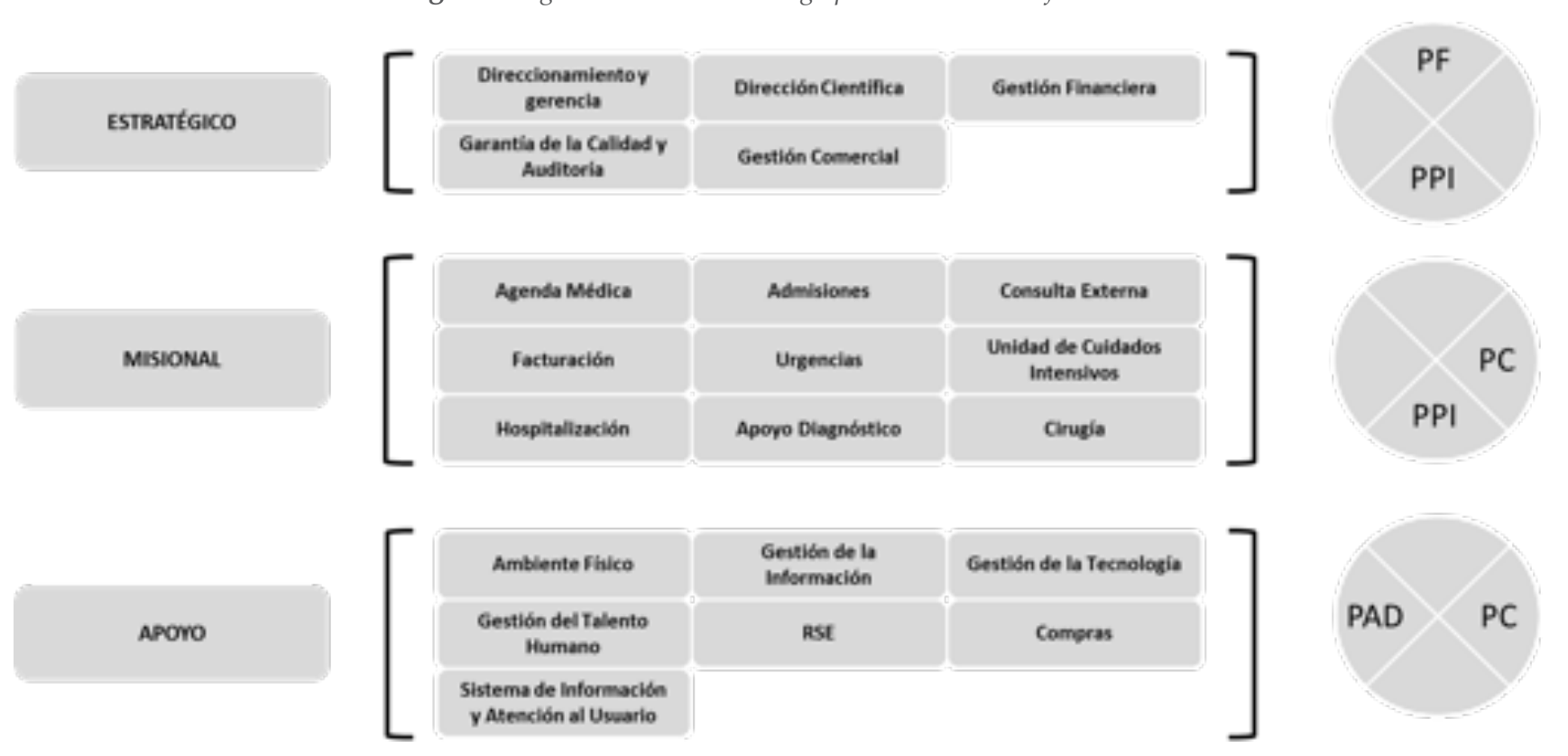
Figura 5. Mapa Estratégico propuesto.

Figure 5. Strategic map proposed.

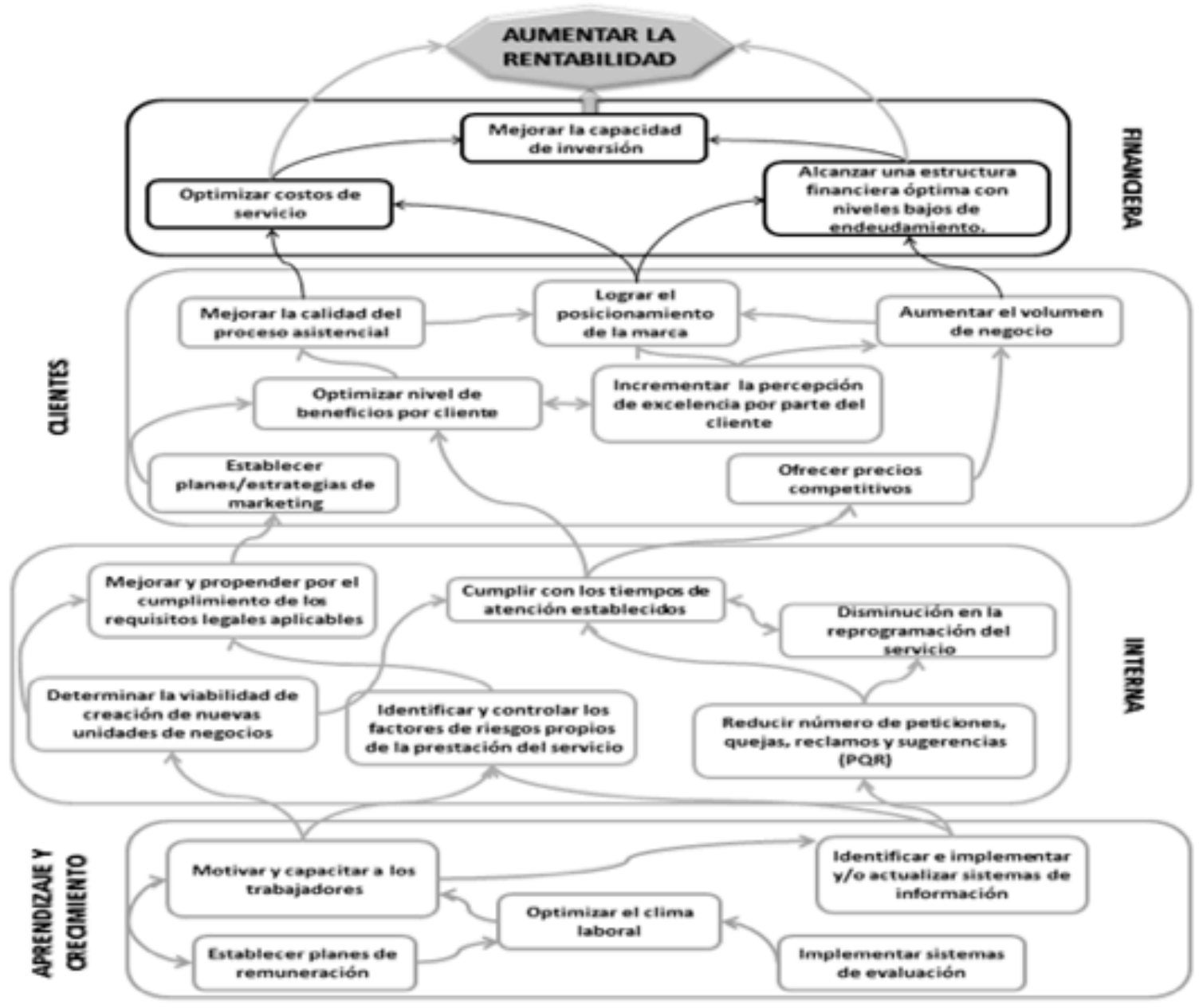

Para efectos ilustrativos, la figura 5 da muestra de la articulación de los objetivos estratégicos definidos para las IPS de alta complejidad de la ciudad de Barranqui1la, a través de un Mapa estratégico en donde a manera de diagrama causa efecto se señalan los vínculos entre objetivos y entre perspectivas a fin de encaminar las planeación de modo más coherente y participativo [21].

\section{CONCLUSIONES}

La población objetivo se encuentra certificada bajo la norma ISO 9001:2008. Sin embargo, la mayoría de las organizaciones no han trabajado en la implementación de Sistemas Integrados de Gestión, que incorporen acciones orientadas hacia la excelencia que demuestren niveles superiores de calidad, garanticen mayor seguridad en la atención y fortalezcan la comunicación con el usuario.

Se desarrolló una propuesta conceptual integrada, en la cual se establece la alineación de los tres modelos, como base fundamental para el diseño del Sistema Integrado de Gestión propuesto para las IPS de alta complejidad de la ciudad de Barranquilla, identificando los aspectos comunes y no comunes, con el fin de estable- cer estrategias que den respuesta a las principales problemáticas presentadas en el sector y que se mejore el funcionamiento de la gestión organizacional.

Se identificaron los factores claves del éxito en las IPS de alta complejidad, con el fin de plantear oportunidades de mejoramiento, dentro de las cuales se destacan la minimización de los costos de no calidad gracias a la intervención, control y seguimiento de cada uno de los procesos, la optimización de los recursos, el posicionamiento en el sector y el aumento de la rentabilidad. Con la articulación de los tres modelos de gestión, se logró diseñar una herramienta de gestión efectiva que permite evaluar la gestión integral de la organización, tanto administrativa como operativamente.

Con base en el diagnóstico que se obtuvo luego de la evaluación de cada organización, se pudo establecer las principales deficiencias que se presentan, logrando identificar como una de las principales falencias el debilitamiento del factor Recursos Humanos, evidenciando la poca motivación y capacitación de los colaboradores de las organizaciones. Aspectos como la formación y satisfacción del personal, temas como poca motivación 
o los métodos de captación y formación de personal debe intervenirse. Por otra parte, la escasa prevención y planificación que se tiene al iniciar las prestaciones del servicio, y el crecimiento igualmente desorganizado que dificultan las gestiones de diferentes tipos de riesgo o impiden contar con una infraestructura acorde a las expectativas de los usuarios.

Se hace necesario implantar un sistema de indicadores que articule la operatividad del proceso con la planeación estratégica de la organización, controlando los procesos claves y permitiendo analizar la gestión en cada unidad funcional.

El seguimiento al servicio prestado y la satisfacción de las necesidades y expectativas del cliente durante la atención, deben constituirse en el pilar fundamental para garantizar la fidelización de los clientes.

Muchas de las organizaciones mostraron gran interés por la implementación del sistema debido a su integralidad, y a que contempla como uno de sus ejes elementales el Sistema de Acreditación en Salud, teniendo en cuenta que son muy pocas las Instituciones de Salud que se encuentran acreditadas en Colombia, y más aún la ciudad de Barranquilla.

\section{REFERENCIAS}

[1] E. Duque, Y. Gómez, “Evolución conceptual de los modelos de medición de la percepción de calidad del servicio: una mirada desde la educación superior", Suma de Negocios, 5 (12), 180-191, 2014.

[2] A. Solano (2017) Informe de Gestión Abril - Junio de 2017 [Internet], Secretaría de Salud de Barranquilla. Disponible desde: http://www.barranquilla.gov.co/salud/index. php/informes / cat_view/125-informes-totales / 25informes / 133-2017> [Äcceso 16 de enero 2018].

[3] M.A. Carmona, E.M. Suárez, A. Calvo-Mora, R. PeriáñezCristóbal, "Sistemas de gestión de la calidad: un estudio en empresas del sur de España y norte de Marruecos", European Research on Management and Business Economics, 22 (2), 8-16, 2016.

[4] J. Álvarez, M. Vila, J.A. Fraiz, M.C. Del Río, "Relación entre herramientas y factores críticos de la calidad", Revista Europea de Dirección y Economía de la Empresa, 23 (1), 82-97, 2014.

[5] M. Gómez, J. Deslaureiers, M. Alzate, Como hacer tesis de maestría y doctorado: Investigación, escritura y publicación. Bogotá: Ecoediciones, 2010, pp. 96-98.

[6] R. Hernández, C. Fernández, P. Baptista, Metodología de la investigación. México: Mc Graw Hill, 2006, pp. 66-67.

[7] C. Arenas, C. Tamayo, "Indicadores administrativos y financieros antes y después de la Acreditación en las Instituciones Prestadoras del Servicio de Salud en Colombia", CES Medicina, 24 (2), 9-20, 2010.

[8] R.S. Kaplan, D.P. Norton, Cómo utilizar el cuadro de mando integral. Barcelona: Gestión 2000, 2001, pp. 87-89.
[9] A. Cadena, L. Giraldo, S. Mora (2010) Evaluación de la satisfacción del cliente interno en la IPS Oral Medic S.A [Internet], repositorio digital Universidad CES. Disponible desde: <http://bdigital.ces.edu.co> [Acceso 9 de Octubre 2012].

[10] C. Alerani, J. Monterde, C. Abelló, “Modelo Europeo de Gestión y Evaluación de la Calidad (EFQM)", El farmacéutico hospitales, 2 (180), 18-30, 2006.

[11] L. Garzón (2008) Mejoramiento del proceso de direccionamiento estratégico con base en los estándares del Sistema Único de Acreditación en salud del sistema obligatorio de garantía de la calidad del SGSSS [Internet], repositorio digital Universidad CES. Disponible desde: <http://bdigital.ces.edu.co $>$ [Acceso 25 de Septiembre 2012].

[12] C. Parra, V. Villa, “Gestión de la calidad con el modelo EFQM en diez (10) PYMES metalmecánicas de Medellín", EIA, 6 (11), 9-19, 2009.

[13] S. Zuñiga, M. Pérez, C. Vargas, “Un Cuadro de Mando Integral para una Empresa del Sector Minero Chileno", Panorama socioeconómico, 42 (29), 44-46, 2011.

[14] H. Gutiérrez, Calidad total y productividad. México: Mc Graw Hill, 2010, pp. 138-140.

[15] S. González, "Sistemas integrados de gestión, un reto para las pequeñas y medianas empresas", Escenarios, 9 (1), 69-89, 2011.

[16] T. Jorgensen, A. Remmen, D. Mellado, "Integrated management systems - three different levels of integration", Journal of Cleaner Production, 14 (8), 713-722, 2006.

[17] M. Carmona (2008) La integración de sistemas de gestión normalizados sobre la base de los procesos [Internet], Instituto Andaluz de Tecnología. Disponible desde: <http:/ / www.iat. es> [Acceso 25 de Junio 2012].

[18] A. Zutshi, A. Sohal, "Integrated management system: The experiences of three Australian organizations", Journal of Manufacturing Technology Management, 16 (2), 211-232, 2005.

[19] J. Muñoz, La Gestión Integrada, Calidad, Seguridad y Medio Ambiente. Pamplona: Serforem, SL, 2004, pp. 65-66.

[20] J.A. Giménez, D. Jiménez, M. Martínez, “La gestión de calidad: importancia de la cultura organizativa para el desarrollo de variables intangibles", Revista europea de dirección y economía de la empresa, 23 (3), 115-126, 2014.

[21] L. Pérez, M. Guillén, A.J. Bañón-Gomis, “Influencia de los factores de contingencia en el desarrollo del cuadro de mando integral y su asociación con un rendimiento mejor. El caso de las empresas españolas", Spanish Accounting Review, 20 (1), 8294, 2017. 